\title{
ARTICLE
}

MULTIPLE MYELOMA GAMMOPATHIES

\section{Disease monitoring with quantitative serum IgA levels provides a more reliable response assessment in multiple myeloma patients}

\author{
Alissa Visram $^{1,2}$ - Iuliana Vaxman $\mathbb{D}^{1,3,4} \cdot$ Abdullah S. Al Saleh $^{1,5} \cdot$ Harsh Parmar $^{6} \cdot$ Angela Dispenzieri $^{1}{ }^{1}$. \\ Prashant Kapoor $^{1}$ - Martha Q. Lacy ${ }^{1}$ - Morie A. Gertz ID $^{1}$ - Francis K. Buadi ID $^{1}$ - Suzanne R. Hayman ${ }^{1}$. \\ David Dingli $\mathbb{D}^{1} \cdot$ Rahma Warsame ${ }^{1} \cdot$ Taxiarchis Kourelis $^{1} \cdot$ Mustaqeem Siddiqui $\mathbb{D}^{1} \cdot$ Wilson Gonsalves $^{1} \cdot$ \\ Eli Muchtar $\mathbb{D}^{1} \cdot$ John A. Lust ${ }^{1} \cdot$ Nelson Leung $^{7}{ }^{7} \cdot$ Robert A. Kyle $^{1} \cdot$ David Murray $^{8}{ }^{8} \cdot$ S. Vincent Rajkumar $^{1} \cdot$ \\ Shaji Kumar (D) ${ }^{1}$
}

Received: 12 October 2020 / Revised: 13 January 2021 / Accepted: 1 February 2021 / Published online: 23 February 2021

(c) The Author(s), under exclusive licence to Springer Nature Limited 2021. This article is published with open access

\begin{abstract}
Unlike IgG monoclonal proteins (MCPs), IgA MCP quantification is unreliable due to beta-migration of IgA MCPs on serum protein electrophoresis (SPEP). The utility of nephelometric quantitative $\operatorname{IgA}$ (qIgA) to monitor IgA multiple myeloma (MM) is unclear. We retrospectively studied disease response kinetics using qIgA versus MCPs by SPEP, and developed and validated novel qIgA disease assessment criteria in 491 IgA MM patients. The SPEP MCP nadir occurred a median of 41 (IQR 0-102) days before the qIgA. The median time to achieve a partial response (PR) was shorter using standard IMWG versus qIgA response criteria (32 vs 58 days, $p<0.001$ ). Stratification by qIgA criteria, unlike IMWG criteria, led to clear separation of the progression-free survival curves of patients achieving a PR or very good PR. There was a consistent trend toward earlier detection of disease progression using qIgA versus IMWG progression criteria. In conclusion, monitoring IgA MM using MCP-based IMWG criteria may be falsely reassuring, given that MCP levels on SPEP decrease faster than qIgA levels. The qIgA response criteria more accurately stratify patients based on the progression risk and may detect disease progression earlier, which may lead to more consistent measurement of trial endpoints and improved patient outcomes.
\end{abstract}

Supplementary information The online version contains supplementary material available at https://doi.org/10.1038/s41375021-01180-x.

Shaji Kumar

kumar.shaji@mayo.edu

1 Division of Hematology, Mayo Clinic, Rochester, MN, USA

2 University of Ottawa, Ottawa Hospital Research Institute, Ontario, Canada

3 Institute of Hematology, Davidoff Cancer Center, Rabin Medical Center, Petah-Tikvah, Israel

4 Israel Sackler Faculty of Medicine Tel-Aviv University, TelAviv, Israel

5 Division of Hematology and HSCT, King Saud bin Abdulaziz University for Health Sciences, Riyadh, Saudi Arabia

6 Division of Hematology, John Theurer Cancer Center at Hackensack University, Hackensack, NJ, USA

7 Division of Nephrology, Mayo Clinic, Rochester, MN, USA

8 Department of Laboratory Medicine and Pathology, Mayo Clinic, Rochester, MN, USA

\section{Introduction}

Multiple myeloma (MM) is an incurable malignancy arising from plasma cells. Neoplastic plasma cells can secrete monoclonal immunoglobulins which are monitored and used as surrogate markers of disease activity. The serum protein electrophoresis (SPEP) is one of the gold standard laboratory methods for quantifying monoclonal proteins (MCPs). While IgG MCPs most commonly migrate in the gamma fraction of the SPEP, IgA MCPs migrate in the beta fraction of the SPEP in $\sim 30-40 \%$ of patients $[1,2]$. Betamigration can lead to an unreliable quantification of the MCP $[1,3,4]$ due to the co-migration of physiologic proteins such as transferrin and complement proteins. Therefore, in the $20-30 \%$ of patients with an IgA isotype of MM [5-8] the electrophoretic migration of MCP may limit the utility of a SPEP derived MCP in disease assessments. Quantitative nephelometric and turbidometric immunoassays have been studied as a means to monitor IgA MCPs [9] given that monoclonal IgA proteins often 
predominate over the small normal polyclonal $\operatorname{IgA}$ background.

The International Myeloma Working Group (IMWG) response criteria [10], which assess disease response and progression based primarily on changes in the MCP measured by SPEP, are the universally accepted standard for assessing disease response and progression in both the clinical and research setting. Due to limitations in SPEP MCP quantification, the IMWG criteria state that in $\operatorname{IgA} \mathrm{MM}$ patients the quantitative $\operatorname{IgA}(\mathrm{q} \operatorname{IgA})$ measurements are preferred for disease assessments. However, this recommendation is based on expert opinion, and the clinical utility of qIgA levels compared with SPEP to assess disease response and prognosticate patient outcomes has not been well studied. Therefore, the aims of this study were to compare the kinetics of disease response in IgA MM patients using both MCP on SPEP and qIgA levels, and to develop and validate response criteria using qIgA.

\section{Patients and methods}

We retrospectively assessed IgA MM patients treated at Mayo Clinic between January 1, 2004 and June 19, 2019. Newly diagnosed MM (NDMM) patients were included in the testing cohort if they were evaluated at Mayo Clinic at MM diagnosis, had serial MCP and qIgA levels available within the first 6 months of diagnosis, and had a qIgA level above the upper limit of normal (ULN) at diagnosis (defined as $\geq 0.365 \mathrm{~g} / \mathrm{dL}$ at our laboratory). Patients were included in the relapsed refractory clinical trial validation cohort (RRMM-CT) if they had relapsed IgA MM with serial qIgA levels available from within 6 months of the start of study therapy and had a qIgA above the ULN at study onset. Demographic, baseline laboratory data, disease response and progression data were abstracted manually from the electronic medical record. This study was approved by the Mayo Clinic Institutional Review Board.

\section{Statistical analysis}

Baseline characteristics in NDMM versus RRMM-CT and comparisons between IMWG versus qIgA responses were compared using the Wilcoxon rank-sum test (for continuous outcomes) or chi-square tests (for dichotomous outcomes). Pearson's correlation test was used to evaluate the association between MCP and qIgA levels. Cohen's kappa statistic was used to assess the concordance between the classification of disease response by IMWG and qIgA response criteria. The Kaplan-Meier method was used for time-to-event analyses. Progression-free survival (PFS) was defined as the time between the initiation of therapy (firstline therapy for the NDMM cohort and clinical trial therapy for the RRMM-CT cohort) and disease progression (as defined by the IMWG criteria) or death. Overall survival (OS) was defined as the time from progression to death. Uni- and multivariable Cox proportional hazards models were used to assess the risk of progression using IMWG or qIgA criteria, adjusting for risk factors associated with progression. A two-sided $p$ value $<0.05$ was considered to be significant. All statistical analyses were performed using JMP Pro v14.1 (SAS Institute, Cary, NC).

\section{Results}

A total of 516 newly diagnosed IgA myeloma patients were identified. On review of the medical charts, patients lacking baseline qIgA levels $(n=12)$ or serial qIgA levels measured at our institution within the first 6 months of diagnosis $(n=$ $205)$, or those with qIgA levels within the normal range at diagnosis $(n=13)$, were excluded. Therefore, 286 patients with newly diagnosed MM were included and used as a testing (NDMM) cohort. A second cohort of relapsed or refractory IgA MM patients treated on various clinical trials was used as validation (RRMM-CT) cohort. A total of 223 patients were identified, however, 18 were excluded because they did not have serial qIgA levels available. Therefore, the RRMM-CT cohort consisted of a total of 205 IgA MM patients.

The median duration of follow-up was 46 (IQR 23-73) months in the NDMM cohort. The median duration of followup for the RRMM-CT cohort was 31.5 (IQR 12-54) months. In the NDMM and RRMM-CT cohorts, the median MCP on SPEP at treatment initiation was 3.3 (IQR 2.4-4.2) g/dL and 2.3 (IQR $1.3-3.2) \mathrm{g} / \mathrm{dL}$, respectively $(p<0.001)$. The median qIgA at diagnosis was 3.1 (IQR 1.9-4.3) g/dL for NDMM cohort and 2.2 (IQR $1.2-3.3$ ) g/dL at study entry in the RRMM-CT cohort $(p<0.001)$. Nineteen out of the 35 NDMM patients without a measurable serum MCP also had no measurable urine MCP or involved FLC (as defined by the IMWG [10]) at diagnosis, however, the median qIgA at diagnosis amongst these 19 patients was elevated at 0.8 (IQR $0.6-1.1) \mathrm{mg} / \mathrm{dL}$. Further baseline characteristics of both cohorts are outlined in Table 1.

The baseline MCP levels (measured by SPEP) correlated poorly with baseline qIgA levels when the MCP was $<1 \mathrm{~g} / \mathrm{dL}$ or $>6 \mathrm{~g} / \mathrm{dL}$, however, the association improved (Pearson's $r=0.857,95 \%$ CI 0.829-0.882) when the MCP was between 1 and $6 \mathrm{~g} / \mathrm{dL}$ (Fig. 1A). As demonstrated in Fig. 1B, the plateau in MCP occured earlier than the plateau in $\mathrm{qIgA}$, rendering serial measurements of $\mathrm{MCP}$ uninformative due to unquantifiable protein levels. Similarly, increases in $\mathrm{q} \operatorname{IgA}$ above the ULN preceded increases in MCP prior to progression. From this we inferred that changes in qIgA levels may more accurately reflect disease burden. Therefore, we 
Table 1 Baseline characteristics of IgA MM patients included in the testing (NDMM) and validation (RRMM-CT) cohorts.

\begin{tabular}{|c|c|c|}
\hline & $\begin{array}{l}\text { NDMM cohort } \\
(N=286)\end{array}$ & $\begin{array}{l}\text { RRMM-CT cohort } \\
(n=205)\end{array}$ \\
\hline Age at diagnosis & $64(59-70)$ & $65(58-71)$ \\
\hline \multicolumn{3}{|l|}{ Gender } \\
\hline Male $-n(\%)$ & $179(63)$ & $120(59)$ \\
\hline Female $-n(\%)$ & $107(37)$ & $85(41)$ \\
\hline \multicolumn{3}{|l|}{ ISS at diagnosis, $n(\%)$} \\
\hline 1 & $64(23)$ & $46(24)$ \\
\hline 2 & $122(43)$ & $103(53)$ \\
\hline 3 & $96(34)$ & $45(23)$ \\
\hline \multicolumn{3}{|l|}{ R-ISS at diagnosis, $n(\%)$} \\
\hline 1 & $41(15)$ & $10(11)$ \\
\hline 2 & $180(68)$ & $73(78)$ \\
\hline 3 & $44(17)$ & $11(12)$ \\
\hline \multicolumn{3}{|l|}{ FISH, $n(\%)$} \\
\hline Standard risk & $164(70)$ & $61(63)$ \\
\hline High risk & $70(30)$ & $36(37)$ \\
\hline \multicolumn{3}{|l|}{ First-line treatment, $n(\%)$} \\
\hline ASCT & $148(52)$ & 77 (104) \\
\hline IMID + PI + steroid & $85(30)$ & - \\
\hline IMID + steroid & $75(26)$ & - \\
\hline PI + Alkylator + steroid & $75(26)$ & - \\
\hline PI + steroid & $21(7)$ & - \\
\hline IMID + Alkylator + steroid & $13(5)$ & - \\
\hline Steroid only & $9(3)$ & - \\
\hline Alkylator + steroid & $6(2)$ & - \\
\hline PI + Anthracycline + steroid & $2(1)$ & - \\
\hline $\begin{array}{l}\text { Median quantitative } \mathrm{qIgA} \text { prior to } \\
\text { treatment, g/dL (IQR) }\end{array}$ & $3.1(1.9-4.3)$ & $2.2(1.2-3.3)$ \\
\hline $\begin{array}{l}\text { Median MCP prior to } \\
\text { treatment, g/dL (IQR) }\end{array}$ & $3.3(2.4-4.2)$ & $2.3(1.3-3.2)$ \\
\hline $\begin{array}{l}\text { Measurable }{ }^{\mathrm{a}} \mathrm{MCP} \text { prior to } \\
\text { treatment, } n(\%)\end{array}$ & $251(88)$ & $160(84)$ \\
\hline Beta-migrating MCP, $n(\%)$ & $122(43)$ & $77(38)$ \\
\hline
\end{tabular}

${ }^{a}$ Measurable serum monoclonal protein at diagnosis is defined at $\geq 1 \mathrm{~g} / \mathrm{dL}$.

developed response criteria using the change in qIgA levels (as defined in Table 2) in order to assess whether stratifying patients based on changes in qIgA levels could better predict disease outcomes.

\section{Rate of response in monoclonal protein and quantitative $\lg A$}

The line plot in Fig. 2A is a visual demonstration of the mean percent change in MCP and $\mathrm{qIgA}$ from baseline during the first four cycles of therapy in the NDMM cohort. It shows that the MCP plateaus even whilst the qIgA continues to decrease. The median time between initiation of first-line treatment and nadir of MCP was 84 (IQR 43-145) days; the median time to nadir qIgA level was 145 (IQR 90-234) days. The onset of MCP nadir occurred at a median of 41 (IQR 0-102) days earlier than the qIgA nadir. At the onset of MCP nadir, 282 patients had qIgA levels measured; the median qIgA level was 0.3 (IQR $0.2-0.6$ ) $\mathrm{g} / \mathrm{dL}$, and $119(40 \%)$ patients had a qIgA level above the ULN $(\geq 0.365 \mathrm{~g} / \mathrm{dL})$. In the 240 patients who reached a MCP nadir of $0 \mathrm{~g} / \mathrm{L}$, the median qIgA level was 0.2 (IQR $0.1-0.4$ ) g/dL and $79(33 \%)$ had a qIgA level above the ULN. Of the 157 patients with a normal qIgA level, 138 had a concurrent serum immunofixation (sIFE) performed, and 106 (77\%) had a detectable IgA MCP on sIFE. Of the 19 patients that had a normal qIgA at MCP nadir but no sIFE tested, 14 (74\%) had abnormalities on the SPEP that were suggestive of an unquantifiable residual MCP. Of the 79 patients with a qIgA above the ULN at MCP nadir, all 64 patients with an accompanying sIFE had a detectable IgA MCP, and 13 patients without a concurrent sIFE had abnormalities noted in the gamma $(n=4)$ or beta $(n=9)$ fractions of the SPEP. All patients achieving a complete response (CR) by IMWG criteria (serum and urine immunofixation showing no MCP, as bone marrow biopsies were not performed in all individuals) had a qIgA level within the normal range, with a median qIgA of 0.06 (IQR 0.03-0.1) g/dL.

During the first four cycles of induction therapy, 108 patients achieved a partial response (PR) by both the IMWG response criteria [10] and the proposed qIgA criteria (Table 2). The median time to achieve PR was significantly shorter if response was assessed using the IMWG criteria compared to the qIgA criteria (32 [IQR 25-56] versus 58 [29-88] days, respectively, with $p<0.001$ ), as seen in Fig. 2B. Similarly, 95 patients achieved a very good partial response (VGPR) by both IMWG and qIgA criteria within the first four cycles of therapy. The respective median time to achieve a VGPR was significantly shorter using the IMWG criteria compared to the $\mathrm{q} \operatorname{IgA}$ response criteria (56 [IQR 33-67] versus 69 [IQR 55-109] days, $p<0.001$ ), as shown in Fig. 2C. Patients with a beta-migrating MCP had a significantly shorter time to achieve IMWG PR (28 vs 35 days, $p<0.001$ ) and IMWG VGPR (44 vs 60 days, $p=0.01$ ) compared to patients with a gamma-migrating MCP.

\section{Defining qlgA response criteria}

\section{Newly diagnosed multiple myeloma (NDMM) cohort}

In order to assess the concordance between the IMWG and qIgA response criteria, the best IMWG response was compared to the best qIgA response (using the nadir qIgA prior to disease progression). The definition of $\mathrm{CR}$ was highly concordant between the two staging systems (kappa $=0.89,95 \%$ CI 0.83-0.94), as shown in Supplementary Table 1 . Nine patients achieved a best response of VGPR by IIgA criteria, and CR by IMWG criteria. The discordant response classification in these nine patients occurred because the qIgA nadir occurred a median of 42 (IQR 32-91) days before the serum immunofixation was 
A)

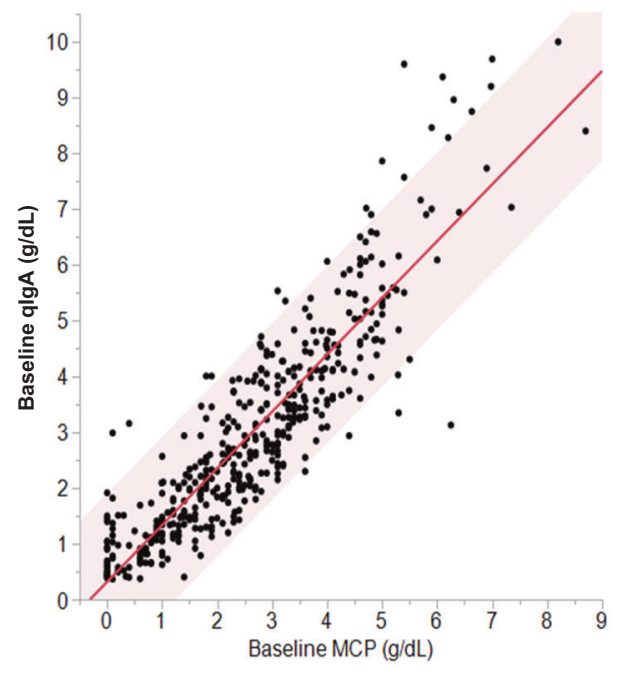

Fig. 1 The association between qIgA and MCP measured by SPEP in the combined NDMM and RRMM-CT cohorts. A The median change in serial qIgA and MCP levels post treatment and prior to IMWG progression are shown, with error bars indicating the respective interquartile ranges. B The correlation of baseline MCP and $\mathrm{qIgA}$ levels collected simultaneously for the combined NDMM and RRMM-
B)

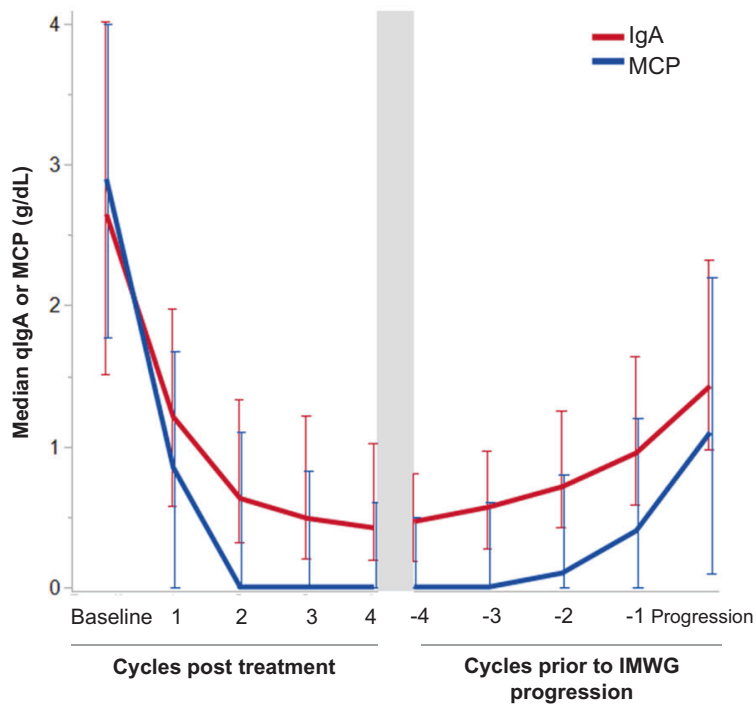

CT cohorts, the line of best fit and $95 \%$ confidence interval are shown in red. The qIgA correlated poorly with MCP when MCP was $<1 \mathrm{~g} / \mathrm{dL}$ (Pearson's $r=0.189,95 \%$ CI -0.033 to -0.394 ) or $>6 \mathrm{~g} / \mathrm{dL}$ (Pearson's $r=0.4,95 \% \mathrm{CI}-0.165$ to 0.768 ), however, the correlation improved when MCP was $1-6 \mathrm{~g} / \mathrm{dL}$ (Pearson's $r=0.857,95 \% \mathrm{CI}$ 0.829-0.882)
Table 2 Response criteria based on quantitative $\operatorname{IgA}$.

\begin{tabular}{|c|c|}
\hline Assessment & Definition \\
\hline \multicolumn{2}{|l|}{ Disease response } \\
\hline Complete response $(\mathrm{CR})$ & qIgA less than the ULN and sIFE negative \\
\hline Very good partial response (VGPR) & $\begin{array}{l}\geq 90 \% \text { decrease in } \text { qIgA level and sIFE positive, OR qIgA below the ULN and } \\
\text { sIFE positive }\end{array}$ \\
\hline Partial response (PR) & $50-89 \%$ decrease in qIgA level \\
\hline Minimal response (MR) & $25-49 \%$ decrease in $\mathrm{qIgA}$ level \\
\hline \multicolumn{2}{|l|}{ Disease progression } \\
\hline $\mathrm{qIgA}_{250}$ progression & $\begin{array}{l}>0.25 \mathrm{~g} / \mathrm{dL} \text { increase in } \mathrm{qIgA} \text { compared to } \mathrm{qIgA} \text { nadir, and a } \geq 25 \% \text { increase in } \\
\mathrm{qIgA} \text { level compared to the qIgA nadir, and a positive serum immunofixation }\end{array}$ \\
\hline $\mathrm{qIg}_{500}$ progression & $\begin{array}{l}>0.5 \mathrm{~g} / \mathrm{dL} \text { increase in } \mathrm{qIgA} \text { compared to } \mathrm{qIgA} \text { nadir, and a } \geq 25 \% \text { increase in } \mathrm{qIgA} \\
\text { level compared to the } \mathrm{qIgA} \text { nadir, and a positive serum immunofixation }\end{array}$ \\
\hline
\end{tabular}

$U L N$ upper limit of normal $(0.356 \mathrm{~g} / \mathrm{dL})$, sIFE serum immunofixation, $q I g A$ quantitative $\operatorname{IgA}$. noted to be negative. The discordance between the IMWG and $\mathrm{qIgA}$ response definitions was most apparent in the stratification of patients achieving a PR. Of the 134 patients that achieved a VGPR by IMWG criteria, 15 (11\%) patients had a discordant response by qIgA criteria, and all 15 were classified as a PR by qIgA criteria.

\section{Disease response assessments using qlgA and IMWG criteria}

\section{Newly diagnosed multiple myeloma (NDMM) cohort}

The median PFS for the overall training cohort was 29.6 (95\% CI 25.5-32.9) months. Stratification of PFS by qIgA response (Fig. 3A) led to a clear separation in the Kaplan-Meier curves for patients achieving a VGPR or PR, as opposed to stratification of PFS by IMWG criteria (Fig. 3B).

In order to understand why patient outcomes were better stratified using the $\mathrm{qIgA}$ criteria compared to IMWG criteria, particularly in patients achieving a VGPR or PR by IMWG criteria, we compared the MCP and qIgA levels based on the electrophoretic migration of MCPs in the gamma versus beta region. In patients achieving a VGPR or PR by IMWG criteria, at diagnosis there was no significant difference in the MCP or qIgA levels in patients with MCPs migrating in the beta versus gamma regions. However, at the onset of IMWG best response of 
A)

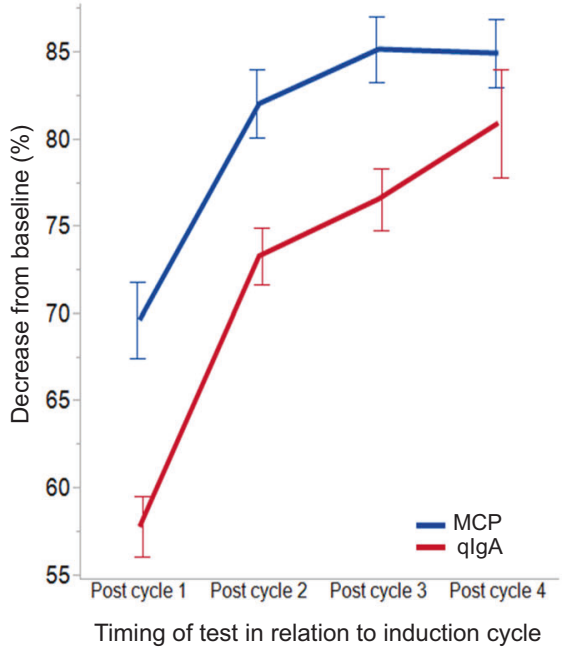

B)

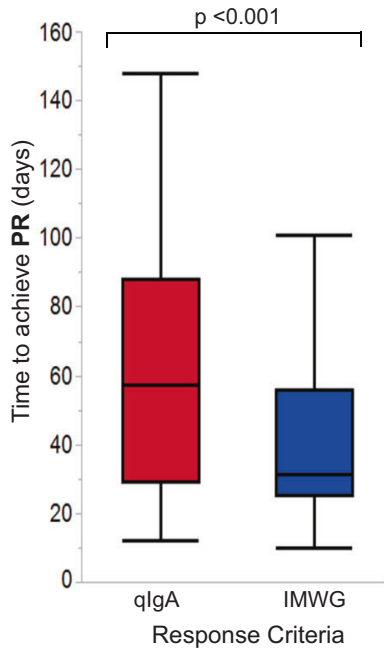

C)

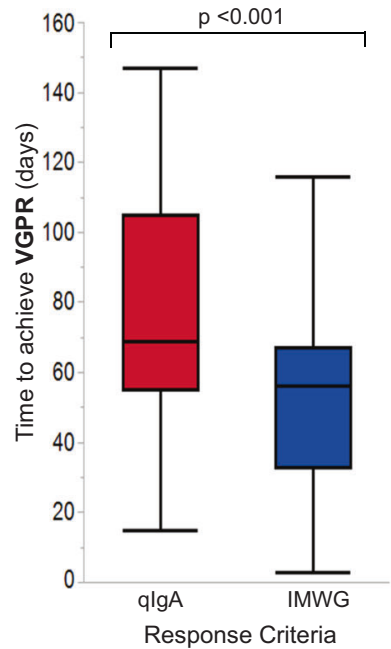

Fig. 2 Response kinetics of the NDMM cohort within the first 4 cycles of induction therapy. A Line plot showing the mean percent decrease in monoclonal protein (MCP) and quantitative $\operatorname{IgA}(\mathrm{qIgA})$ compared to baseline. Patients meeting criteria for progression were excluded. Standard error is indicated by the vertical bars. Box plots comparing the median time to $\mathbf{B}$ partial response, or $\mathbf{C}$ very good partial response applying both the qIgA and IMWG response criteria.
A) NDMM Cohort, stratified by qlgA Response Criteria

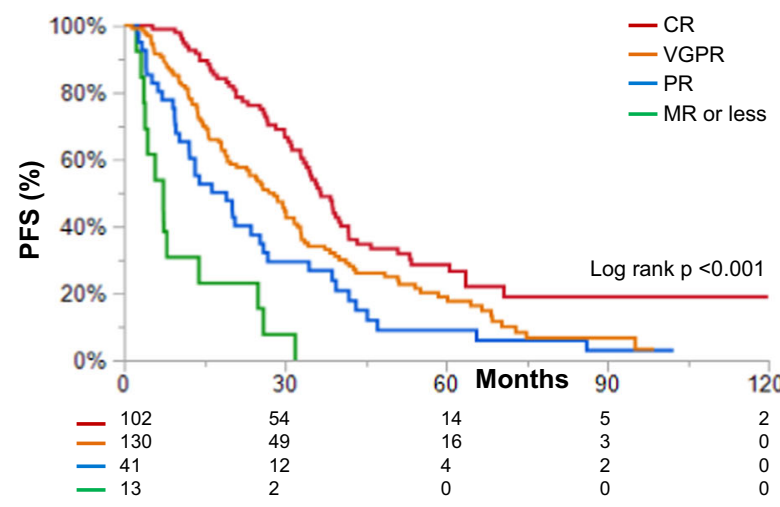

C) RRMM-CT Cohort, stratified by qlgA Response Criteria

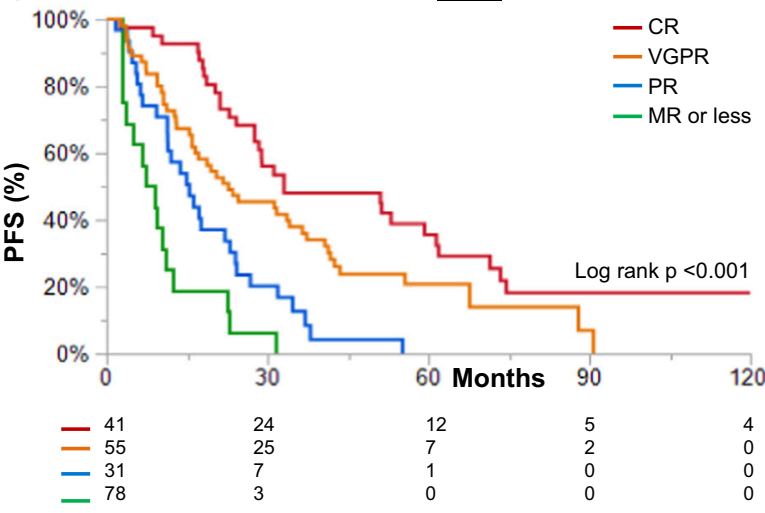

Fig. 3 Progression free survival (PFS) stratified by IMWG and qIgA response criteria. The PFS, stratified by the $\mathrm{qIgA}$ criteria, is shown for the newly diagnosed multiple myeloma (NDMM) testing cohort (A) and relapsed refractory clinical trial (RRMM-CT)
B) NDMM Cohort, stratified by IMWG Response Criteria

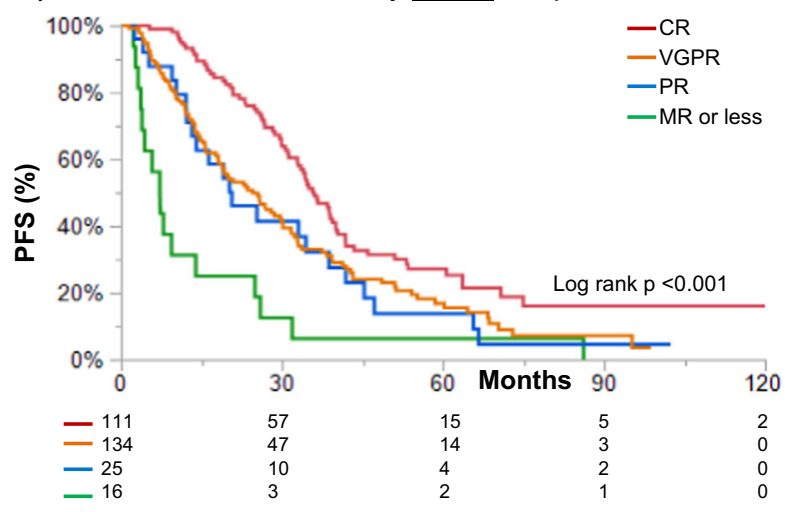

D) RRMM-CT Cohort, stratified by IMWG Response Criteria

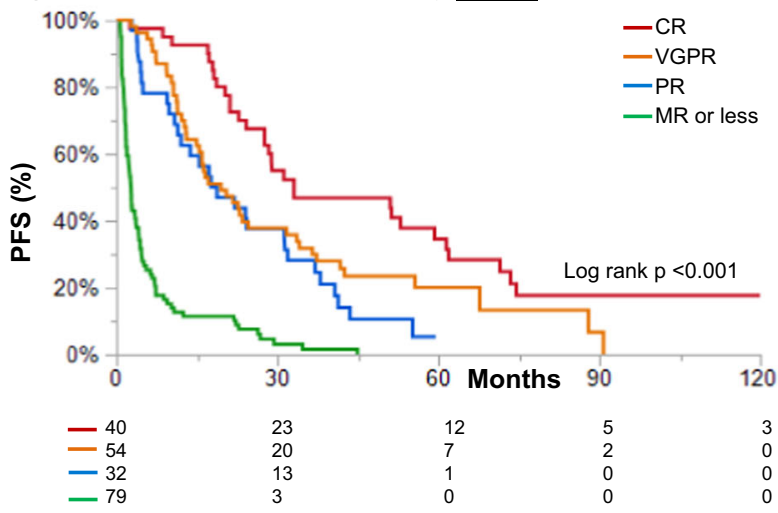

validation cohort $(\mathbf{C})$. In contrast, the PFS stratified by IMWG response criteria is shown for the testing cohort (B) and validation cohort (D). CR complete response, VGPR very good partial response, $\mathrm{PR}$ partial response, MR minimal response. 
VGPR/PR, the median $\mathrm{MCP}$ was $0 \mathrm{~g} / \mathrm{dL}$ regardless of whether the MCP was migrating in the beta or gamma region of the SPEP, but the median qIgA was significantly higher in patients with a beta-migrating MCP compared to a gamma-migrating MCP $(0.41 \mathrm{~g} / \mathrm{dL}$ versus $0.27 \mathrm{~g} / \mathrm{dL}$, respectively, $p=0.013$ ).

On univariable analysis, attaining a best response of VGPR or better reduced the risk of progression when using either the $\mathrm{qIgA}(\mathrm{HR} 0.46,95 \%$ CI $0.34-0.64, p<0.001)$ or IMWG (HR 0.55, 95\% CI 0.39-0.79, $p<0.001$ ) response criteria, when compared to attaining less than a VGPR. However, attaining a CR/VGPR by qIgA criteria was still prognostic of the risk of progression (HR 0.59, 95\% CI $0.40-0.86, p=0.006)$ even after adjusting for age at diagnosis $(\geq 65$ verses $<65$ ), R-ISS at diagnosis (stage 3 versus stage 1 or 2 ), measurable MCP at diagnosis (MCP $\geq 1 \mathrm{~g} / \mathrm{dL}$ versus $<1 \mathrm{~g} / \mathrm{dL}$ ), electrophoretic migration of MCP (gamma versus beta region on SPEP), and ASCT status (those that received ASCT versus those that did not). Importantly, there was no significant decrease in risk of progression in patients achieving a VGPR or better by the IMWG criteria after adjusting for the same covariates (HR 0.80, 95\% CI $0.52-1.23, p=0.309$ ).

\section{Relapsed refractory multiple myeloma patients on clinical trial (RRMM-CT cohort)}

Progression free survival, stratified by disease response, was assessed in the RRMM-CT cohort. The median PFS for the whole RRMM-CT cohort was 12.9 (95\% CI 10.2-17.6) months. The qIgA response criteria were applied to patients in the clinical trial cohort, and the PFS of this cohort was assessed by stratifying patients according to the qIgA criteria (Fig. 3C) or IMWG criteria (Fig. 3D). This analysis validated the finding that the qIgA response criteria was better able to stratify the PFS outcomes in patients achieving a PR or VGPR when compared to the IMWG criteria.

\section{Disease progression assessment using qlgA and IMWG criteria}

\section{Newly diagnosed multiple myeloma (NDMM) cohort}

A total of $209(70 \%)$ patients in the NDMM cohort (the training cohort) met criteria of disease progression by IMWG criteria, with a median MCP of 0.7 (IQR $0-1.9$ ) g/dL and qIgA level of $1.0(0.5-1.8) \mathrm{g} / \mathrm{dL}$ at progression. Of these patients, $78(37 \%)$ had no reported MCP on SPEP at the time of progression (21 had new symptomatic bone lesions, 57 met criteria for light chain progression), despite the fact that $41(53 \%)$ of the 78 patients had a qIgA level above the ULN. More importantly, of the 21 patients progressing due to a new symptomatic bone lesion or plasmacytoma in the absence of a detectable MCP, 14 (67\%) had a qIgA above the ULN.

In order to assess whether qIgA levels could be used to detect progression earlier than MCP levels, we assessed the time to progression using two different qIgA definitions. We defined $\mathrm{qIg}$ A progression as a $\geq 25 \%$ increase in $\mathrm{qIgA}$ level compared to the qIgA nadir, and a positive serum immunofixation, and either an absolute increase in qIgA by $0.25 \mathrm{~g} / \mathrm{dL}$ (the " $\mathrm{qIg} \mathrm{A}_{250}$ " definition) or $0.5 \mathrm{~g} / \mathrm{dL}$ (the "qIgA $\mathrm{A}_{500}$ " definition), as outlined in Table 2. At $\mathrm{qIgA}_{250}$ and $\mathrm{qIgA}_{500}$ progression, the median MCP was 0.2 (IQR $0-0.5) \mathrm{g} / \mathrm{dL}$ and $0.4(0-0.8) \mathrm{g} / \mathrm{dL}$, respectively.

We sought to use time-to-event analyses to compare the time to progression with IMWG versus qIgA criteria. Of the 209 patients meeting the IMWG progression criteria, we excluded 63 patients due to a lack of available serial qIgA levels prior to IMWG criteria-based progression, and 41 patients that did not meet criteria for qIgA progression due to a $<25 \%$ increase in $\mathrm{qIgA}$ at IMWG progression. Therefore, in the NDMM cohort, $105(50 \%)$ patients met criteria for both IMWG and qIgA 250 progression, whereas $89(43 \%)$ patients met criteria for IMWG and $\mathrm{qIgA}_{500}$ progression. The median time to progression was significantly shorter using the $\mathrm{qIgA}_{250}$ criteria definition compared to the conventional IMWG progression criteria (median time to progression of 21 versus 28.6 months, respectively, $p=0.02$ ), as shown in Fig. 4A. Though there was a trend toward earlier assessment of progression with the use of $\mathrm{qIg}_{500}$ criteria compared to IMWG criteria (Fig. 4B), this did not reach statistical significance (median time to progression of 22.1 versus 26.8 months, respectively, $p=0.06$ ). The median OS from IMWG progression date was significantly shorter than from $\mathrm{qIgA}_{250}$ progression date (33.3 versus 49.8 months, $p=0.019$ ), and there was a trend toward shorter median OS from IMWG progression date compared to $\mathrm{qIgA}_{500}$ progression date (33.3 versus 52.8 months, $p=0.057)$.

\section{IgA multiple myeloma patients on clinical trial}

In the RRMM-CT validation cohort, 158 (76\%) patients met criteria for IMWG progression, and at progression the median MCP was 1.4 (IQR 0.3-2.4) g/dL and median qIgA was 1.6 (IQR $0.8-2.6) \mathrm{g} / \mathrm{dL}$. Thirty-two (20\%) of patients had a MCP of $<0.5 \mathrm{~g} / \mathrm{dL}$ at progression, however, the median $\mathrm{qIgA}$ at IMWG progression in these patients was 0.6 (IQR 0.2-0.8) g/dL. Thirteen patients met the IMWG criteria for progression due to new bone lesions or plasmacytoma in the absence of a measurable MCP, and the qIgA was elevated above the ULN in $8(62 \%)$ of these patients.

In order to assess whether the $\mathrm{qIg}_{250}$ and $\mathrm{qIgA}_{500}$ progression criteria could predict progression earlier than 


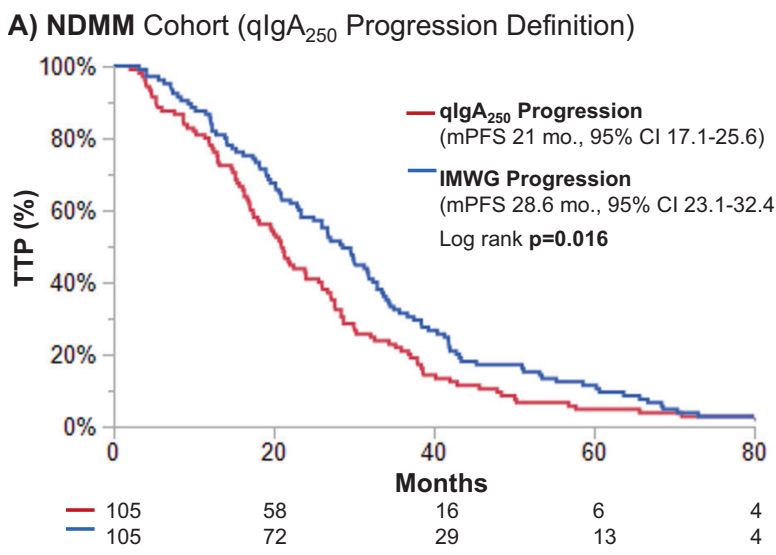

C) RRMM-CT Cohort ( lg $_{250}$ Progression Definition)

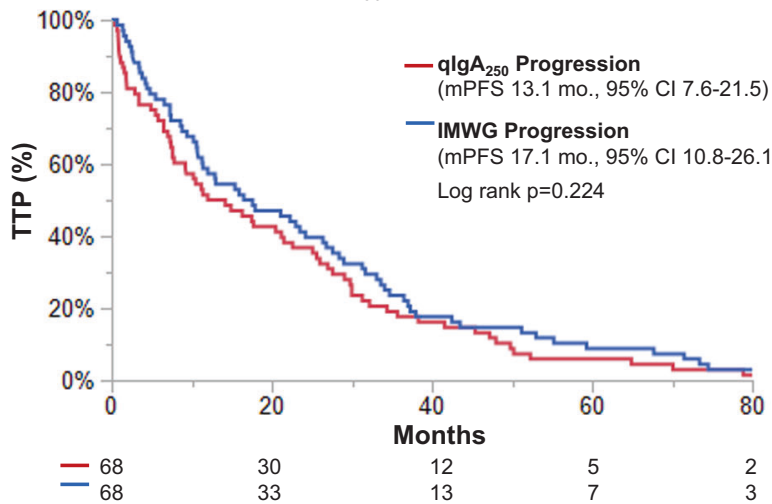

Fig. 4 The time to progression (TTP) using the IMWG progression criteria versus the qIgA progression criteria was compared. The TTP was assessed by IMWG progression criteria versus the $\mathrm{qIgA}_{250}$ progression criteria $(\mathbf{A})$, and $\mathrm{q}^{\operatorname{Ig} \mathrm{A}_{500}}$ progression criteria $(\mathbf{B})$ in the newly diagnosed multiple myeloma (NDMM) testing cohort.

IMWG criteria, patients meeting both qIgA and IMWG progression criteria were included in the time-to-event analysis. Of the 158 patients meeting criteria for IMWG progression, 68 did not have serial qIgA measurements prior to IMWG progression, and 22 were excluded because they did not meet criteria for $\mathrm{qIgA}_{250}$ progression. In the remaining 68 patients who progressed by both the IMWG and $\mathrm{qIgA}_{250}$ criteria, there was a trend toward an earlier assessment of progression with $\mathrm{qIgA}_{250}$ criteria (Fig. 4C) with a median time to progression of 13.1 months by $\mathrm{qIgA}_{250}$ criteria versus 17.1 months by IMWG criteria ( $p=$ 0.224). Similarly, in the 52 patients progressing by both $\mathrm{qIgA}_{500}$ and IMWG criteria, the trend toward earlier progression with qIgA criteria was seen, however, this was not statistically significant (Fig. 4D). There was a trend toward a shorter median OS from the date of IMWG progression compared to the $\mathrm{qIgA}_{250}$ progression date (45.9 versus 64.1 months, $p=0.080$ ), and from the date of IMWG progression compared to the $\mathrm{qIg}_{500}$ progression date (45.9 versus 63 months, $p=0.226$ ).
B) NDMM Cohort (qlgA $\mathrm{A}_{500}$ Progression Definition)

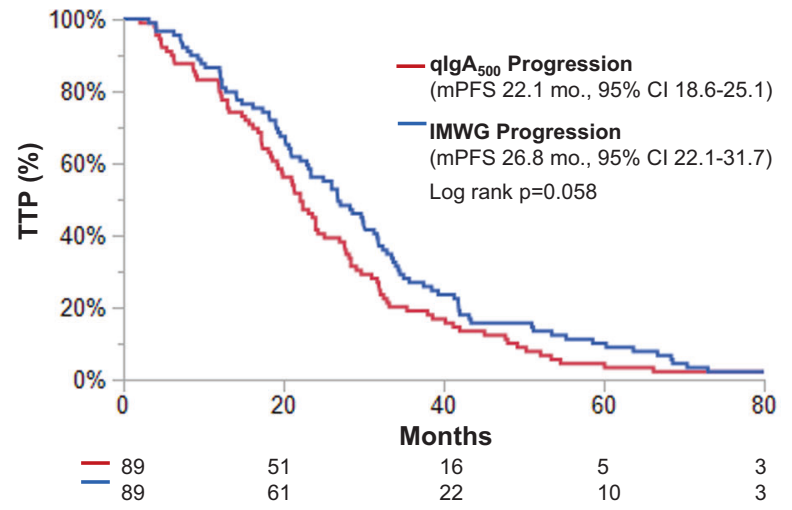

D) RRMM-CT Cohort (qlgA ${ }_{500}$ Progression Definition)

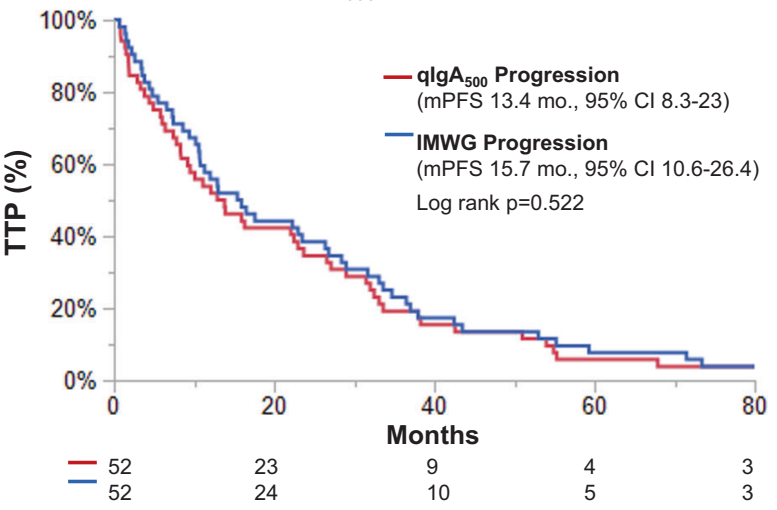

Similarly, the TTP was assessed using the IMWG progression criteria compared to the $\mathrm{qIgA}_{250}$ progression criteria (C) and $\mathrm{qIgA}_{500}$ progression criteria (D) in the relapsed refractory clinical trial (RRMMCT) validation cohort.

\section{Discussion}

In this study, we showed that the inaccurate quantification of MCP by SPEP directly affects the clinical response assessment in IgA MM patients. We demonstrate that the artifactual decline in MCP levels overstates response as compared to qIgA levels. Quantitative IgA levels are elevated in $30 \%$ of patients even if MCP levels are undetectable, indicating active disease. Furthermore, at the time of IMWG progression $~ 50 \%$ of patients with no detectable MCP on SPEP had qIgA levels above the ULN. In both the NDMM and RRMM-CT cohorts in this study, 60\% of patients progressing with new symptomatic bone disease had no detectable MCP yet had qIgA levels above the ULN. These findings highlight the shortcomings of relying solely on SPEP quantification of paraproteins in $\operatorname{IgA} \mathrm{MM}$ and underscore the importance of monitoring patients with MM using qIgA levels. The serial use of qIgA may also allow earlier detection of disease, which may in turn lead to earlier intervention and an improved prognosis in $\operatorname{IgA}$ MM 
patients. Furthermore, as patients with unmeasurable disease are often excluded from clinical trials, routine incorporation of $\mathrm{qIgA}$ levels into clinical trial inclusion criteria will increase the eligibility of IgA MM patients and thereby improve access to novel therapeutic agents. Our study applied the qIgA response criteria only to IgA MM patients with a baseline qIgA above the ULN $(\geq 0.365 \mathrm{~g} / \mathrm{dL})$. Therefore, for ease of implementation, we suggest that measurable disease be defined as a $\mathrm{qIgA} \geq 0.5 \mathrm{~g} / \mathrm{dL}$ (this cutoff would include $98 \%$ of our NDMM cohort and $96 \%$ of our RRMM-CT cohort).

While the depth of disease response has consistently been shown to prognosticate disease outcomes [11-13], our study showed that stratification of patients by IMWG criteria was not as predictive of the risk of progression when compared to qIgA response criteria, particularly in patients achieving a best response of VGPR or PR. Accurate categorization of disease response is imperative in both clinical trial and routine patient care settings. In clinical trials, the depth of response is commonly used as a study endpoint or as eligibility criteria for randomization to further lines of therapy $[14,15]$. Therefore, inaccurate disease response classification may deleteriously affect interpretation of study results. The use of additional lines of therapy to deepen disease response and improve PFS has been demonstrated in the induction and consolidation treatment settings [15-19]. While we do not necessarily advocate altering management to deepen response, it is evident that response classification post treatment influences physicians to provide patients with further lines of therapy for this purpose. In the BMT CTN 0702 trial, 15\% of patients had $\geq 2$ lines of therapy pre-transplant, and the median time to registration was 5 (range 2-14) months, suggesting that patients with suboptimal disease response may have received additional therapy [20]. In this context, in IgA MM patients the use of IMWG response criteria may be falsely reassuring to physicians, which may lead to less aggressive management of disease.

Multiple studies have demonstrated that patients with IgA MM have an unfavorable prognosis compared to their non-IgA MM counterparts [21, 22]. However, retrospective studies assessing the gene expression profiles [8], and cytogenetics [23-26] of MM patients have not reported consistent differences between IgA versus non-IgA MM subtypes to explain the difference in prognosis. Given our study's findings, we propose that the adverse prognosis of IgA MM patients may be in part due to differences in disease management due to misclassification of disease response, or delayed detection of disease due to underestimation of tumor burden.

Previous studies have demonstrated faster response kinetics and deeper IMWG responses in $\operatorname{IgA}$ MM compared to non-IgA MM patients [27, 28], however, the biological basis of these observations were not described. We showed that in IgA MM patients with undetectable MCPs at best response, those with MCPs migrating in the beta fraction of the SPEP had significantly higher $\mathrm{qIgA}$ levels compared to patients with gamma-migrating MCPs. This illustrates that for beta-migrating MCPs, the proportion of MCP quantified is underestimated due to comigration with physiologic proteins. The significant heterogeneity in the methodology used to quantitate betamigrating MCPs between laboratories [3] likely increases the variation in MCP measurements between centers, and further limits the applicability of response criteria that rely on SPEP quantification. Though beta-migrating paraproteins can be more accurately quantified using immunoglobulin heavy/light chain assays $[1,29]$ or mass spectrometry [30, 31], these methods are not widely available in clinical practice.

Although we did not show statistically significant reductions in the time-to-disease progression using qIgA progression versus IMWG progression criteria, this is likely due to the small number of patients who progressed in our study. There was a consistent trend that using qIgA progression criteria led to earlier detection of progression compared to IMWG progression. Further studies are needed to assess whether implementation of qIgA response and progression criteria leads to improvements in disease outcomes. One could also extrapolate that monitoring $\mathrm{qIgA}$ levels in patients with smoldering $\mathrm{MM}$ or monoclonal gammopathy of undetermined significance could lead to detection of disease progression earlier, prior to the onset of end-organ damage. Even though the use of qIgA may be more relevant for patients with beta-migrating peaks, it is not easy for community oncologists to make these decisions on a case by case basis, and hence we believe that all qIgA patients could be followed by qIgA. Another important implication of our findings is the use of response criteria for assessment in clinical trials. Depth of response and time-toevent analysis are important endpoints in the efficacy readout of clinical trials and routine use of the $\operatorname{IgA}$ for response assessment will allow for consistent assessment of these endpoints.

In conclusion, this study showed that in IgA MM patients the decline in serum MCP levels may be falsely reassuring, as MCP levels decrease faster than qIgA levels. These findings reinforce the IMWG recommendations to use qIgA levels to monitor disease response in IgA MM patients. The proposed and validated qIgA response criteria better stratify patients based on the risk of progression compared to IMWG criteria and may allow for earlier detection of disease progression, which may in turn lead to earlier intervention and improved outcomes for IgA MM patients. 


\section{Compliance with ethical standards}

Conflict of interest The authors declare no competing interests.

Publisher's note Springer Nature remains neutral with regard to jurisdictional claims in published maps and institutional affiliations.

Open Access This article is licensed under a Creative Commons Attribution 4.0 International License, which permits use, sharing, adaptation, distribution and reproduction in any medium or format, as long as you give appropriate credit to the original author(s) and the source, provide a link to the Creative Commons license, and indicate if changes were made. The images or other third party material in this article are included in the article's Creative Commons license, unless indicated otherwise in a credit line to the material. If material is not included in the article's Creative Commons license and your intended use is not permitted by statutory regulation or exceeds the permitted use, you will need to obtain permission directly from the copyright holder. To view a copy of this license, visit http://creativecommons. org/licenses/by/4.0/

\section{References}

1. Boyle EM, Fouquet G, Guidez S, Bonnet S, Demarquette H, Dulery R, et al. IgA kappa/IgA lambda heavy/light chain assessment in the management of patients with $\operatorname{IgA}$ myeloma. Cancer. 2014;120:3952-7.

2. Turner KA, Frinack JL, Ettore MW, Tate JR, Graziani MS, Jacobs JFM, et al. An international multi-center serum protein electrophoresis accuracy and M-protein isotyping study. Part I: factors impacting limit of quantitation of serum protein electrophoresis. Clin Chem Lab Med. 2020;58:533-46.

3. Tate JR, Keren DF, Mollee P. A global call to arms for clinical laboratories - Harmonised quantification and reporting of monoclonal proteins. Clin Biochem. 2018;51:4-9.

4. Rieckenberg M, Collier C, Raymond M, Matthews J. Monitoring of monoclonal gammopathies: rational use of densitometry and rate nephelometry. Clin Biochem. 1994;27:457-61.

5. Fine JM, Lambin P. Distribution of heavy chain classes and light chain types in 757 cases of monoclonal gammapathies. Biomedicine. 1975;23:323-7.

6. Kyle RA, Gertz MA, Witzig TE, Lust JA, Lacy MQ, Dispenzieri A, et al. Review of 1027 patients with newly diagnosed multiple myeloma. Mayo Clin Proc. 2003;78:21-33.

7. Schaar CG, le Cessie S, Snijder S, Franck PF, Wijermans $\mathrm{PW}$, Ong C, et al. Long-term follow-up of a population based cohort with monoclonal proteinaemia. $\mathrm{Br} \mathrm{J}$ Haematol. 2009;144:176-84.

8. Nair B, Waheed S, Szymonifka J, Shaughnessy JD Jr, Crowley J, Barlogie B. Immunoglobulin isotypes in multiple myeloma: laboratory correlates and prognostic implications in total therapy protocols. Br J Haematol. 2009;145:134-7.

9. Murray DL, Ryu E, Snyder MR, Katzmann JA. Quantitation of serum monoclonal proteins: relationship between agarose gel electrophoresis and immunonephelometry. Clin Chem. 2009;55:1523-9.

10. Kumar S, Paiva B, Anderson KC, Durie B, Landgren O, Moreau $\mathrm{P}$, et al. International Myeloma Working Group consensus criteria for response and minimal residual disease assessment in multiple myeloma. Lancet Oncol. 2016;17:e328-e46.

11. van de Velde HJ, Liu X, Chen G, Cakana A, Deraedt W, Bayssas M. Complete response correlates with long-term survival and progression-free survival in high-dose therapy in multiple myeloma. Haematologica. 2007;92:1399-406.

12. Harousseau JL, Attal M, Avet-Loiseau H. The role of complete response in multiple myeloma. Blood 2009;114:3139-46.

13. Vij R, Kumar S, Zhang MJ, Zhong X, Huang J, Dispenzieri A, et al. Impact of pretransplant therapy and depth of disease response before autologous transplantation for multiple myeloma. Biol Blood Marrow Transpl. 2015;21:335-41.

14. Moreau P, Attal M, Hulin C, Arnulf B, Belhadj K, Benboubker L, et al. Bortezomib, thalidomide, and dexamethasone with or without daratumumab before and after autologous stem-cell transplantation for newly diagnosed multiple myeloma (CASSIOPEIA): a randomised, open-label, phase 3 study. Lancet. 2019;394:29-38.

15. Ladetto M, Pagliano G, Ferrero S, Cavallo F, Drandi D, Santo L, et al. Major tumor shrinking and persistent molecular remissions after consolidation with bortezomib, thalidomide, and dexamethasone in patients with autografted myeloma. J Clin Oncol. 2010;28:2077-84.

16. Jackson GH, Davies FE, Pawlyn C, Cairns DA, Striha A, Collett $\mathrm{C}$, et al. Response-adapted intensification with cyclophosphamide, bortezomib, and dexamethasone versus no intensification in patients with newly diagnosed multiple myeloma (Myeloma XI): a multicentre, open-label, randomised, phase 3 trial. Lancet Haematol. 2019;6:e616-e29.

17. Cavo M, Pantani L, Petrucci MT, Patriarca F, Zamagni E, Donnarumma $\mathrm{D}$, et al. Bortezomib-thalidomide-dexamethasone is superior to thalidomide-dexamethasone as consolidation therapy after autologous hematopoietic stem cell transplantation in patients with newly diagnosed multiple myeloma. Blood. 2012;120:9-19.

18. Leleu X, Fouquet G, Hebraud B, Roussel M, Caillot D, Chretien ML, et al. Consolidation with VTd significantly improves the complete remission rate and time to progression following VTd induction and single autologous stem cell transplantation in multiple myeloma. Leukemia. 2013;27:2242-4.

19. Fouquet G, Hebraud B, Garciaz S, Stoppa AM, Roussel M, Caillot $\mathrm{D}$, et al. Partial response at completion of bortezomibthalidomide-dexamethasone (VTd) induction regimen upfront in multiple myeloma does not preclude response to VTd in consolidation. J Cancer. 2014;5:248-52.

20. Stadtmauer EA, Pasquini MC, Blackwell B, Hari P, Bashey A, Devine $\mathrm{S}$, et al. Autologous transplantation, consolidation, and maintenance therapy in multiple myeloma: results of the BMT CTN 0702 trial. J Clin Oncol. 2019;37:589-97.

21. Usmani SZ, Hoering A, Cavo M, Miguel JS, Goldschimdt H, Hajek R, et al. Clinical predictors of long-term survival in newly diagnosed transplant eligible multiple myeloma-an IMWG Research Project. Blood Cancer J. 2018;8:123.

22. Greipp PR, Miguel JS, Durie BGM, Crowley JJ, Barlogie B, Bladé J, et al. International staging system for multiple myeloma. J Clin Oncol. 2005;23:3412-20.

23. Fonseca R, Blood E, Rue M, Harrington D, Oken MM, Kyle RA, et al. Clinical and biologic implications of recurrent genomic aberrations in myeloma. Blood. 2003;101:4569-75.

24. Yadav P, Merz M, Mai EK, Försti A, Jauch A, Goldschmidt H, et al. Cytogenetic aberrations in multiple myeloma are associated with shifts in serum immunoglobulin isotypes distribution and levels. Haematologica. 2018;103:e162-e4.

25. Wang L, Jin FY, Li Y, Sun JN, Zhang JJ, Tang R, et al. IgA type multiple myeloma, clinical features, and prognosis. Chin Med J. 2018;131:1249-50.

26. Muddasani R, Ho A, Akerman M, Islam S, Polak M, SuarezLondono JA, et al. Association between immunoglobulin isotypes 
and cytogenetic risk groups in multiple myeloma. Blood. 2018;132:5585.

27. Caillon H, Attal M, Moreau P, Dejoie T. Multiple myeloma (MM): impact of immunoglobulin isotype on the speed of response. Blood. 2015;126:4191.

28. Binder M, Rajkumar SV, Gertz MA, Lacy MQ, Dispenzieri A, Buadi FK, et al. Predictors of early response to initial therapy in patients with newly diagnosed symptomatic multiple myeloma. Am J Hematol. 2015;90:888-91.
29. Katzmann JA, Willrich MA, Kohlhagen MC, Kyle RA, Murray DL, Snyder MR, et al. Monitoring IgA multiple myeloma: immunoglobulin heavy/light chain assays. Clin Chem. 2015;61:360-7.

30. Mills JR, Kohlhagen MC, Dasari S, Vanderboom PM, Kyle RA, Katzmann JA, et al. Comprehensive assessment of M-proteins using nanobody enrichment coupled to MALDI-TOF mass spectrometry. Clin Chem. 2016;62:1334-44.

31. Keren DF, Schroeder L. Challenges of measuring monoclonal proteins in serum. Clin Chem Lab Med. 2016;54:947-61. 